This item was submitted to Loughborough's Research Repository by the author.

Items in Figshare are protected by copyright, with all rights reserved, unless otherwise indicated.

\title{
Improving the reliability of busbar protection system with IEC 61850 GOOSE based communication
}

PLEASE CITE THE PUBLISHED VERSION

https://doi.org/10.1007/978-3-319-68324-9_50

\section{PUBLISHER}

Springer, Cham (@ Springer International Publishing AG)

\section{VERSION}

AM (Accepted Manuscript)

\section{PUBLISHER STATEMENT}

This work is made available according to the conditions of the Creative Commons Attribution-NonCommercialNoDerivatives 4.0 International (CC BY-NC-ND 4.0) licence. Full details of this licence are available at: https://creativecommons.org/licenses/by-nc-nd/4.0/

\section{LICENCE}

CC BY-NC-ND 4.0

\section{REPOSITORY RECORD}

Bogdanon, Dimitar, Georgi Dimitrov, and Francisco M. Gonzalez-Longatt. 2019. "Improving the Reliability of Busbar Protection System with IEC 61850 GOOSE Based Communication". figshare. https://hdl.handle.net/2134/26975. 


\title{
Improving the Reliability of Busbar Protection System with IEC 61850 GOOSE Based Communication
}

\author{
Dimitar Bogdanov ${ }^{1}$, Georgi Dimitrov ${ }^{2}$ and \\ Francisco Gonzalez-Longatt ${ }^{3}$ \\ ${ }^{1}$ Technical University of Sofia, Bulgaria, dbogdanov@tu-sofia.bg \\ 2 TriEl Ltd. Company, Sofia, Bulgaria, georgi.st.dimitrov@gmail.com \\ ${ }^{3}$ Loughborough University, F.Gonzalez-Longatt@lboro.ac.uk
}

\begin{abstract}
The main task of the "relay" protection system is to provide trip commands in case of faults and to prevent the operation of the electrical equipment in dangerous modes. The set of the protection functions and the system structure have an impact on the reliability and respectively dependability of the protected structure. In some smaller node-point electrical facilities like step-up substations for PVPPs and wind-farms, the set of protection functions required by the respective regulations can be limited. The multifunctional features of new generations of Intelligent Electronic Devices (IEDs) and the options to use IEC 61850 based communication, protection and automation, provide opportunities for improvement of the busbar protection system.
\end{abstract}

Keywords: relay protection, busbars, reliability, IED, IEC 61850.

\section{Introduction}

The contemporary grid protection and automation schemes are becoming more and closer as structure and communication topology to data exchange in computer networks. The exchange of information is widely made via Ethernet based networks, in some cases using WEB interfaces. The optical links are based also practically on the hardware and software common for the data exchange in computer networks. This utilizes the implementation of universal hardware and software, but imposes questions related to cyber security, vulnerability to unauthorized access, software "bugs", etc.

The implementation of IEC 61850 features for substation automation provides many opportunities to improve the functionality of the respective protection system. For the particular study, experiments were made to implement a function for busbar protection utilizing the functionality of the outgoing power lines protections.

\section{Improving the Relay Protection System Functionality}

The contemporary operated electrical grids are challenged by the high penetration of renewable sources of electrical power generation [1,2]. The node points for connec- 
tion of renewables - typically Photo Voltaic Power Plants (PVPPs) and wind farms are sometimes regarded as non-vital for the integrity of the grid. With the increase of the concentrated capacity of such generating facilities, and their general share in the total electrical power generation mix, the importance of the reliability of the protection schemes for such node points increases. For the particular study, a model was made for a simple HV busbar system with simulated internal (on the busbar system) fault and external one - in the outgoing power lines. By utilization of IEC 61850 GOOSE (Generic Object Oriented System Event) message, the directional ground fault overcurrent protection was used for busbar protection logic for accelerated tripping of faults recognized "inside" the substation zone $[9,10,11]$. In such manner the classical bus differential function (ANSI 87) can be realized not with dedicated central unit and distributed measurement points at the bus connected feeders, but with data exchange between the feeder protection devices. Definitely, the proposed solution is not regarded as replacement of the bus differential protection, but as option to realize additional protection of node point. The proposed approach is to have instantaneous response.



Fig. 1. Experimental test scheme.

The tests were made with two IEDs connected with fiber-optic cables and Ethernet link to exchange GOOSE messages, for the operation of the ground fault protection. In this experimental setting were used Line Differential Protection IEDs. In this case the IED used fiber-optic to transmit differential type of data (like current, voltage, 
frequency etc.) with the opposite device. For GOOSE communication between the IEDs is used Ethernet channel (the scheme assumes two IEDs installed on two outgoing feeders in one substation). The "busbar voltage" was supplied to both devices from the respective analog outputs of the testing device. Two main groups of experiments were made - one with current supplied to the protection devices flowing "straight" for both and another when the current is fed in "reversed" direction to one of the IEDs.



Fig. 2. Display of the IED.

In Fig. 2 is presented the measurement of the line protection IED in the case of currents injected as presented in Fig. 3, in order to simulate internal busbar fault. The two directional overcurrent functions both recognize "fault in the back" with respect to the power lines and the generated tripping commands are correct (selective).

With this scheme of exchange of GOOSE message between the IEDs, when both relays pick-up, but "fault in the back" is recognized, fault within the zone of the busbar system is identified. Such scheme can help to form busbar protection function (or with some compromise in the physical basis of the operation "artificial differential function"). 


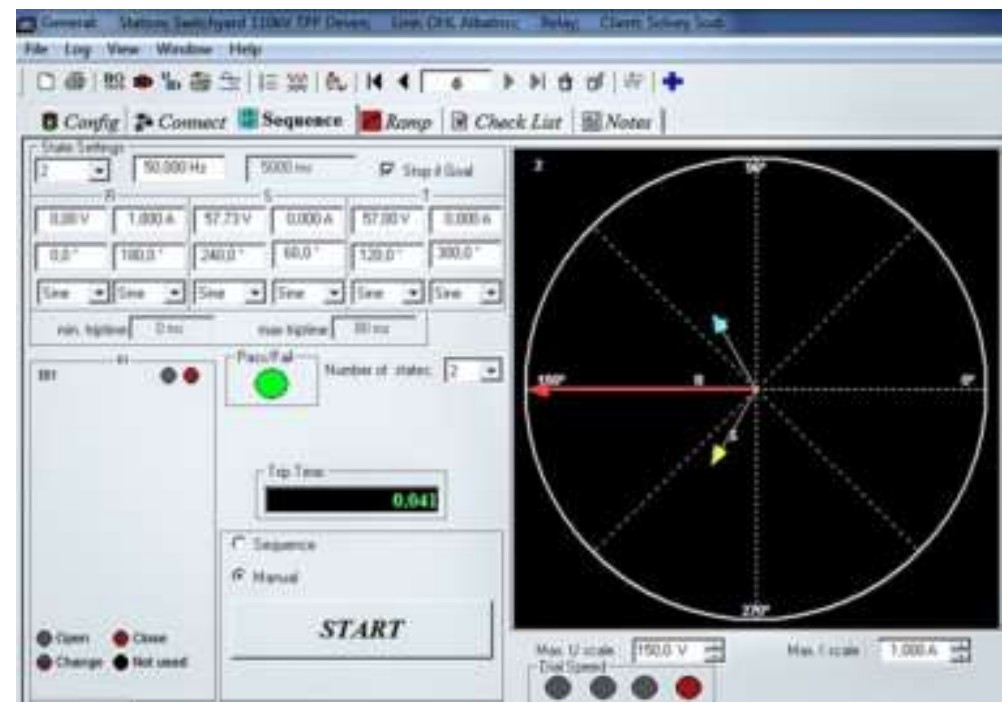

Fig. 3. Testing device signals phasor view.

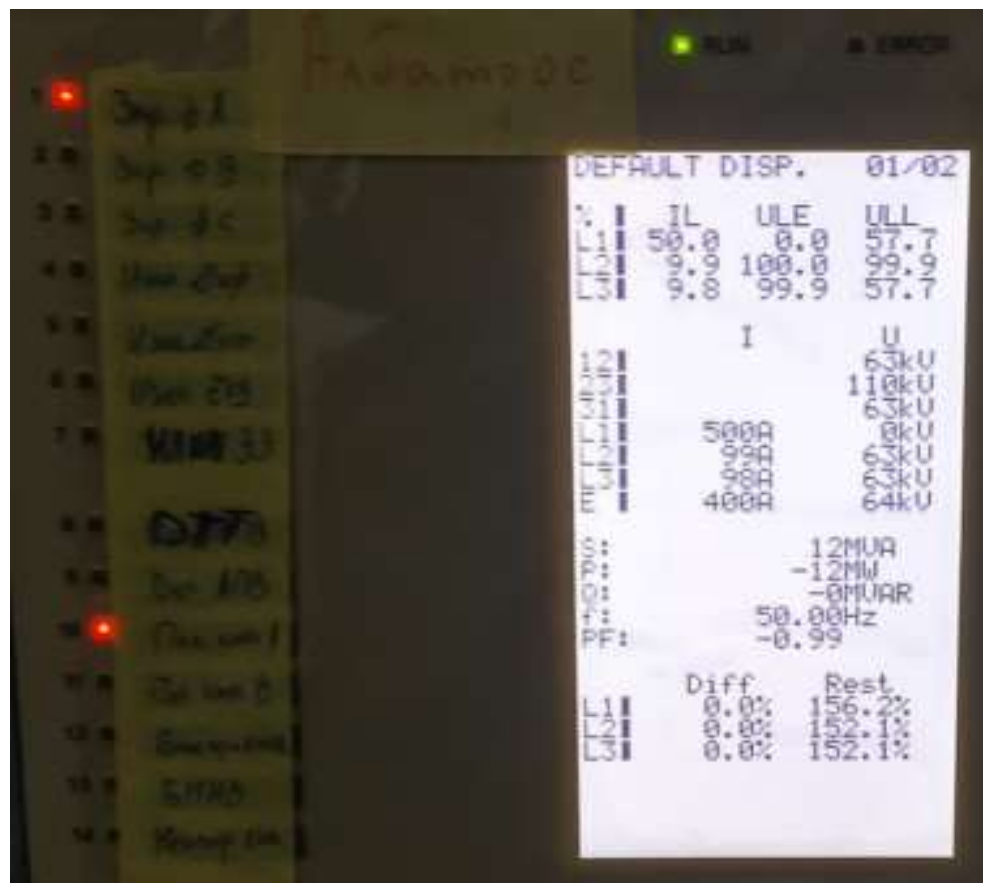

Fig. 4. Display of the IED.

With this scheme of exchange of GOOSE message between the IEDs, when both relays pick-up, but "fault in the back" is recognized, fault within the zone of the bus- 
bar system is identified. Such scheme can help to form busbar protection function (or with some compromise in the physical basis of the operation "artificial differential function").

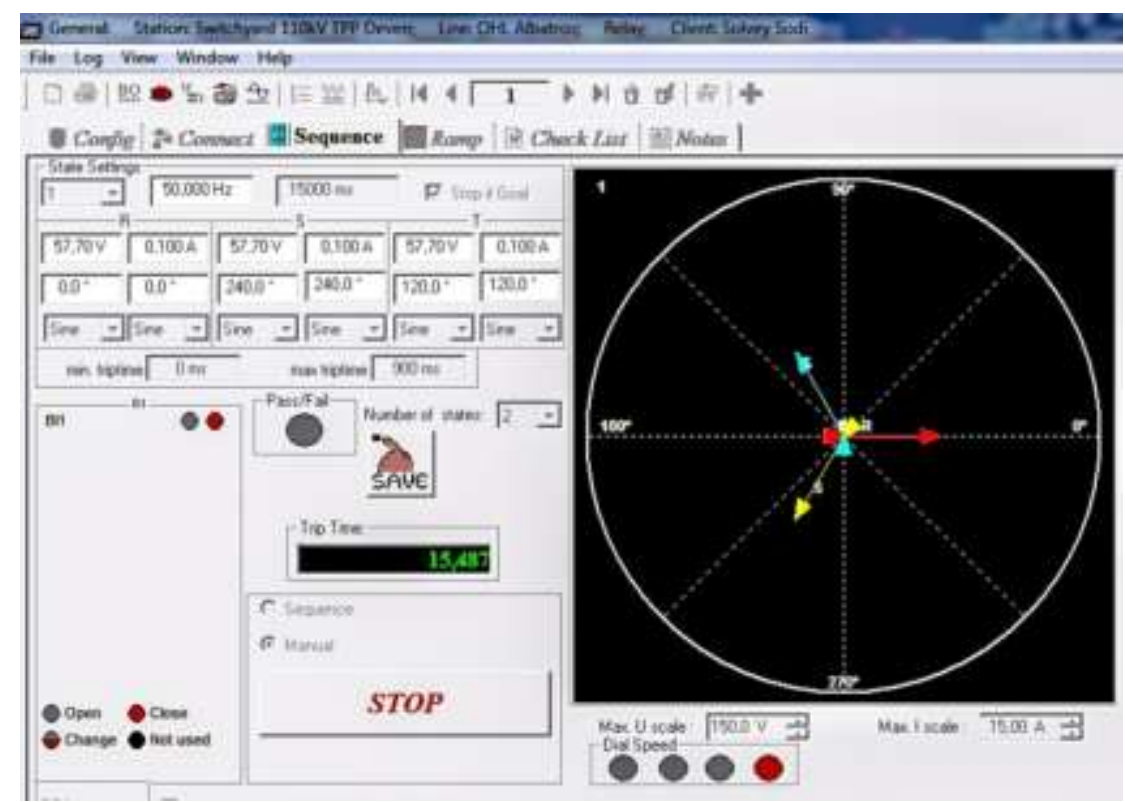

Fig. 5. Testing device signals phasor view.

The proposed scheme operation was tested for the case of fault occurring along one of the power lines [4,6]. In Fig. 4 the behavior of the IED, which has received a blocking signal for ground fault recognized "towards the line" from the other IED, in order not to provide erroneous tripping function is presented. The primary currents were physically simulated with electronic IED testing device, directly connected to the respective analog signal inputs. The IEDs providing protection of the power line correctly generated trip signal, and the other IED was blocked by the received GOOSE message, that the fault current flows "outwards" the busbar system. The tested principle can give improvement of the protection system functionality for small substations, or "node points".

In relation to detection of single phase ground faults, the scheme is applicable for solid grounded networks. For resonance earthed or isolated star point networks, the scheme would be applicable for two or three phase short circuits, based on overcurrent element + directional criterion.

For solidly (effectively) grounded neutral networks with sufficient fault current to be selectively recognized for not being a load current, the power flow could be sufficient as criteria, but for low values of ground fault currents the complexly accounted criteria:

$$
\begin{aligned}
& 3 \mathrm{I}_{0}=\dot{\mathrm{I}}_{\mathrm{A}}+\dot{\mathrm{I}}_{\mathrm{B}}+\dot{\mathrm{I}}_{\mathrm{C}} \\
& 3 \mathrm{U}_{0}=\dot{\mathrm{U}}_{\mathrm{A}}+\dot{\mathrm{U}}_{\mathrm{B}}+\dot{\mathrm{U}}_{\mathrm{C}}
\end{aligned}
$$


The specific angles characterizing the zero sequence power flow $\varphi_{\left(\mathrm{S}_{0}\right)}$ shall be taken into account as well. Precise measurement of the residual voltage and current would be necessary for resonance earthed or isolated star point networks.

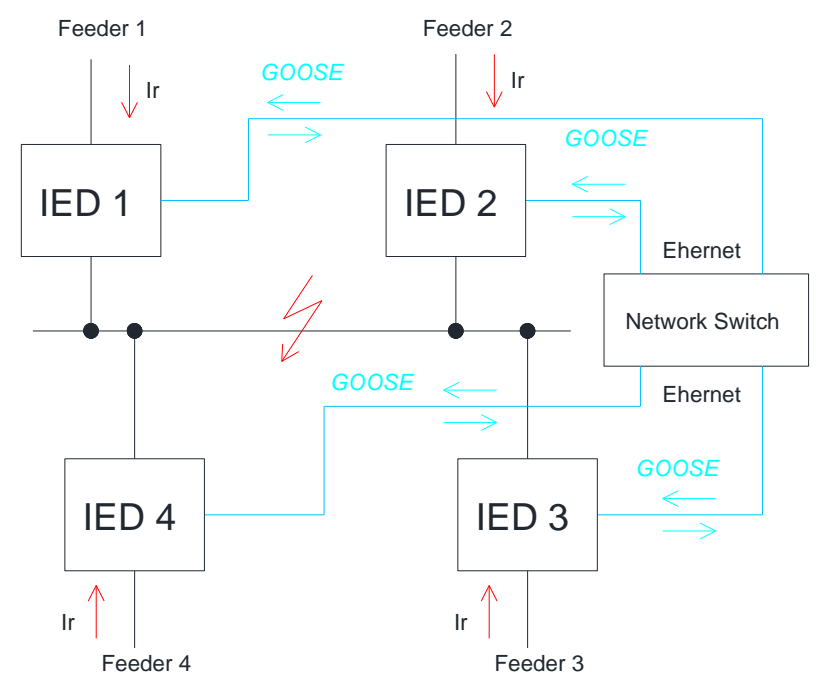

Fig. 6. Scheme to realize a bus protection for busbar with 4 feeders.

As limitation condition for the proposed scheme the specifics of the overcurrent protection used as basis shall be counted.

The typical setting of ground fault function (ANSI 67/67N), which practically is used to form busbar protection "artificial differential" function is calculated as follows:

$$
I_{\text {pick-up }}=\frac{\kappa_{s} \cdot \kappa_{r e s t} \cdot \kappa_{s h}}{\kappa_{v} . \kappa_{m}} I_{m . l .}
$$

where:

$I_{m . l .}$ - maximum permissible load current;

$\kappa_{s}$ - security factor;

$\kappa_{\text {rest }}$ - factor of restart of induction machines;

$\kappa_{v}$ - reset factor of the overcurrent functions;

$\kappa_{m}$ - current transformer ration;

$\kappa_{s h}$ - scheme coefficient;

In this case for determination of $I_{\text {pick-up }}$ is taken the maximum permissible normal current, which can be fed from the respective feeder to the bus section, at the worst in 
this case current distribution. The trip delay of the proposed bus protection function shall be coordinated with the settings of the outgoing feeders.

The fact that as initial "sensitive" element an overcurrent function is used, imposes some limitations towards the sensitivity of the functionality for low imbalanced currents, that may occur in case of high resistance faults in the busbar zone. If high start currents of induction machines have to be taken into account, this will make the scheme even more non-sensitive to high resistance faults in the protected zone. Exchange of GOOSE messages with measured values via the local Ethernet based network can help to improve the sensitivity, but still the delay of the scheme may be higher than dedicated bus differential (ANSI 87) protection scheme. On other hand ultra-fast 87 function may impose overloading on the breakers, as the tripping will be in "relatively early" transient conditions, with significant DC component in the fault current.

\section{Comparison of the Proposed Logic for Bus Protection with Available on the Market Industrial Products}

The leading companies provide two typical schemes for bus differential protection:

"Centralized bus differential protection" - configuration with one central module (without bay units). All CTs signals are routed to one panel - "Busbar protection", comprising the housing of the IED for centralized protection. The central unit collects also binary signal for position status of the respective commutation devices in the switchgear bays. This is the "classical" configuration, used in past for electromechanical and solid state devices. The producers use this concept in contemporary design IEDs $[14,18]$ and the tendency is in the near future significant percent of world producers of "relay" protections to continue to use it. In this scheme there are no separate differential protection bay units as in "Distributed bus differential protection", which means that bigger reliability can be expected from the "hardware". As a disadvantage can be outlined, that when prophylaxis performed for some bays could be more difficult to render safe. Another disadvantage is the big length of copper cables and potential issues with cabling failures.

"Distributed bus differential protection" - the structure is based on the following concept: for every switchgear bay a separate bus differential bay unit is foreseen (peripheral module). The bay unit receives the signals from the CTs, position signals for commutation equipment, etc. and transmits the data to central processing module, typically via fiber optic cable. The main logic is in the central module. When fault is recognized, commands are distributed to the peripheral (bay) modules. This concept is applied in some of the new digital protection structures $[13,15,17]$. The distributed IEDs concept provides flexible functionality and reduction of conventional instrumental copper cables is achieved. The disadvantage of this concept is the higher cost and some aspects of complexity of maintenance. 
Exists one innovation variant [16], which represents that in every IED (like Overcurrent protection, Distance protection etc.) the bay control functionality, needed for differential protection function is integrated. In such design the Central module, which forms the bus differential protection is in permanent communication mode via dedicated channel with the IEDs for the respective bays. This concept is a variant to "Distributed bus differential protection", but costs significantly less, as there are no special separate peripheral modules.

The proposed solution can be used as bus protection system, for places where the "classical" bus differential protection is not obligatory.

The provide solution can help realize fast responding and cost effective protection scheme, without dedicated hardware.

The proposed scheme can be used also as back-up of the bus differential function.

\section{Conclusions}

The provided application of GOOSE messages to form busbar protection function (of ground faults) on the basis of the power line protection IEDs is applicable for small "node points" on the grid, where the busbar differential protection realized with dedicated hardware may not be obligatory. Application of the proposed scheme can be realized in step-up substations of PVPPs, wind farms, small distribution substations in HV grids, etc., [3,7].

In general, the provided solution can be realized with auxiliary contacts of the protection devices and conventional cables, but this will increase the complexity of the scheme and will reduce its reliability. Electromagnetic compatibility issues, galvanic separation benefit and simplicity of the design can be achieved by utilization of fiberoptic based communication [8]. The IEC 61850 GOOSE based communication and respectively acceleration / interlocking gives flexibility, noise immunity, and integrated solution [3,12]. External events can be identified and register for transmission of data to higher level protection / grid control system. Some "external factors" still impede on some substations preferable utilization of shielded Ethernet cables than optics. These obstacles result of the necessity of more precise cable tracing, protection of rodents, some maintenance issues.

The obtained results give justification for the opportunity of GOOSE exchange IEC 61850 implementation in order to obtain reliable bus protection.

The proposed approach can give positive financial effect for investment designs, as additional functionality can be gained, without extra investments for high-cost equipment.

\section{References}

1. Alexander Apostolov. Integration of distributed energy resources. PACWorld magazine. Issue September 2013, pp 18-25. 
2. Jorge Velez, S. Ward, D. Elizondo, System Integrity Protection System (SIPS) PACWorld magazine. Issue March 2014, pp 38-43.

3. Adamiak, M., Baigent, D., Mackiewicz, R., 2009, IEC 61850 Communication Networks and Systems In Substations, 61-68.

4. Application manuals for SIPROTEC Protection Relays, SIEMENS.

5. Buyer's Guide Busbar differential protection IED REB 670, ABB, 2005.

6. Fernandes, C., Borkar, S., Gohil, J., 2014, Testing of Goose Protocol of IEC61850 Standard in Protection IED, 30-35, International Journal of Computer Applications.

7. Falk, H., 2008 IEC 61850-90-5 - an Overview.

8. Fries, S., Seewald, M., 2008, Information security for energy automation: IEC $62351-$ Challenges and Solutions, Magazine for the energy industry.

9. Hoyos, J., Dehus, M., Brown, T., 2012, Exploiting the GOOSE Protocol: A Practical Attack on Cyber - infrastructure, 1508-1513, University of Colorado, USA.

10. Kriger, C., Behardien, S., Retonda-Modiya, J., 2013, A Detailed Analysis of the GOOSE Message Structure in an EIC 61850 Standard - Based Substation Automation System, University of Technology South Africa, Cape Town.

11. Kush, N., Ahmed, E., Branagan, M., Foo, E., 2014, Poisoned GOOSE: Exploiting the GOOSE Protocol, 17-22, Queensland University of Technology.

12. Seeley, N., 2008, Automation at Protection Speeds: IEC 61850 GOOSE Messaging as a Reliable, High - Speed Alternative to Serial Communications, Spokane, Washington.

13. ABB Switzerland Ltd Power Systems. Distributed busbar protection REB500 including line and transformer protection Product Guide.

14. ABB Power Technologies AB, Substation Automation Products Technical reference manua. Busbar differential protection IED REB 670.

15. Siemens AG, Dept. EV S PSN, D-13623 Berlin, Germany. SIPROTEC Manual, Distributed Busbar/Breaker Failure Protection 7SS52.

16. SIPROTEC 5 Low-Impedance Busbar Protection 7SS85.

17. Protecta Electronics Ltd,. Hungary. Distributed busbar protection OGYD manuals.

18. Protecta Electronics Ltd,. Hungary. Centralized busbar protection DGYD manuals. 